\title{
Do Mutual Funds Attract the Right Investor? A Stochastic Dominance Approach
}

\author{
${ }^{*}$ Yudhvir Seetharam, Christo Auret \\ University of the Witwatersrand, South Africa \\ Yudhvir.Seetharam@wits.ac.za
}

\begin{abstract}
Decision theory is concerned with identifying values and uncertainties in a given decision that result in the optimal outcome (Wald, 1939). It is one of the core aspects of any financial or investment decision. We consider the case where the investor has the choice between a passive index (such as a market index) and an actively managed mutual fund. Our analysis aims to determine which option an investor will choose based on a statistical ranking method known as stochastic dominance. We then evaluate this choice against the background information supplied by the mutual fund to ascertain whether the choice given by stochastic dominance is indeed in line with the investor profile given by the mutual fund. It is found that of the 11 mutual funds examined over the sample period of April 2006 to April 2013, only 4 attract the correct type of investor, 3 attract a mixture of investors and 4 attract (arguably) the wrong type of investor.
\end{abstract}

Keywords: Stochastic Dominance, Mutual Funds, Risk appetite, emerging markets

\section{Introduction}

Consider an investor who has the option to invest in domestic equity. This can be achieved through (broadly) one of three means: investing in an index or Exchange Traded Fund (ETF), investing in a mutual fund, or through individual equity selection. The typical investor would either follow a particular approach or create a portfolio through a combination of the above. We consider the case where the investor has the choice between a passive index (such as a market index) and an actively managed mutual fund. Our analysis aims to determine which option an investor will choose based on a statistical ranking method known as stochastic dominance. We then evaluate this choice against the background information supplied by the mutual fund to ascertain whether the choice given by stochastic dominance is indeed in line with the investor profile given by the mutual fund. This research was motivated by prior work of Seetharam and Auret (2013), where we developed an investment strategy that combined elements of fundamental, technical and behavioural analysis to create a high performing "Fusion" Fund. Apart from tests for robustness, the next intuitive question would be to examine the type of investor that would theoretically invest in our Fusion Fund. As such, the results that followed spurred further investigation into comparisons between other, already existing mutual funds in South Africa. This study will proceed as follows. An overview of the literature surrounding this field and its direct relations will be examined in Chapter 2. Thereafter, Chapter 3 outlines the fusion strategy and appropriate statistical methodology for analysis of its returns. Chapter 4 presents and discusses the results along with particular sensitivity tests and biases that were present in this study. Lastly, Chapter 5 provides an excursus into the caveats and avenues for future research of this study, ending with a conclusion. Appendix A provides mathematical derivations for select components of the statistical methodology, Appendix B provides additional graphs of stochastic dominance tests and Appendix C details the results of the fusion strategy against an (artificial) equally weighted passive benchmark.

\section{Literature Review}

Active and passive portfolio management: A simple question lies at the heart of both finance academia and practice - can a portfolio manager achieve superior returns than the market, after costs? A plethora of studies have been conducted, and yet there has been no clear consensus of whether active or passive portfolio management is more beneficial in terms of returns. Underlying the question is the theory surrounding the EMH. Whilst it is not the purpose of this review to provide an extensive discourse on EMH literature, a brief discussion is instructive. Kendall (1953) analysed a sample of firms from the United Kingdom and found that no autocorrelation was apparent in share prices. This implied that prices behaved in a random manner, with equally likely probabilities of increasing, decreasing or remaining the same. Roberts (1959) enhanced this implication by analysing shares in the United States. Using these two 
findings, Fama (1965) presented the Efficient Markets Hypothesis. Since its publication, the EMH has generated much debate amongst academics and practitioners alike. To date, whilst there may be a consensus in some circles, there is no definitive proof of whether the EMH holds.

The forms of market efficiency: The EMH requires that agents have rational expectations (that is, on average, the population of agents are correct, even when no single agent is) and that these agents update their expectations whenever new information arises. The EMH requires that investors' reactions follow a Gaussian distribution so that no abnormal profits can be realised. Each of the forms of efficiency, as described by Fama (1965) requires a differing set of requirements to hold true. The weak form of market efficiency states that future prices cannot be predicted by analysing past prices. In the long run, investment strategies will not earn excess returns after costs. More specifically, strategies focused on technical analysis will not be able to consistently produce excess returns whereas strategies focused on fundamental analysis may still provide excess returns. Statistically, share prices do not exhibit serial correlation - they follow a random walk. The semi-strong form of market efficiency provides that share prices adjust quickly to public information. Neither a fundamental- nor a technical analysis-based strategy will earn excess returns. However, those that have access to private information may be able to obtain superior returns. Lastly, under strong form efficiency, share prices fully reflect both public and private information. Thus, no sustainable superior returns, after costs, can be achieved in the long run. There is a vast amount of literature surrounding tests for market efficiency. This study indirectly offers a test of the semi-strong and weak forms of efficiency as it utilises financial statement analysis in its methodology.

An alternative view of efficiency: The EMH asserts that financial markets are informationally efficient. That is, one cannot consistently achieve returns in excess of average market returns on a risk-adjusted basis, given the information publicly available at the time the investment is made. Tests of the EMH have yielded both positive and negative results. Inevitably, once a particular result is found, it becomes prone to criticism, sometimes rightfully so. For example, Malkiel (2005) states irrevocably that a passive index fund has significantly beaten an active fund over a 30 year horizon. From this the author concludes that the EMH holds. Malkiel (2005) however fails to mention that transaction costs have not been taken into account - an important factor in deciding which strategy is superior. Roberts (1959) is one of the early academics to suggest enhancing technical analysis with the aid of fundamental analysis. At a time predating the publication of both the EMH and the Capital Asset Pricing Model (CAPM) of Sharpe (1964), the suggestions of Roberts (1959) show that irrespective of whether one perceives the market to be efficient, one can still economise time in the search for greater returns.

Lo (2004 and 2005) describes a new form of market theory - the Adaptive Market Hypothesis (AMH). This approach utilises concepts from finance and the principles of evolution. It is simply stated as follows: "Prices reflect as much information as dictated by the combination of environmental conditions and the number and nature of 'species' in the economy" (Lo, 2005, p. 19). Species refer to market participants (asset managers, hedge funds, traders, inter alia). Thus, the efficiency of the market at any point in time is related to the factors of evolution and competition present. It presents a simple, philosophical and pleasantly intuitive view of market efficiency. Market efficiency can be seen as cyclical. There are times of inefficiency and efficiency. For a market to become efficient, it must first be inefficient and vice versa. The influence of market participants (through trading or financial product innovation) influences this efficiency, sometimes in a disruptive way. To date, no formal methodology has been published on testing the AMH. However, authors have nonetheless proposed and tested methods (for example, Todea, Ulici \& Silaghi, 2009; inter alia). It is with this viewpoint (in support of the AMH), that this study draws upon.

The role of stochastic dominance in empirical finance: Decision theory is concerned with identifying values and uncertainties in a given decision that result in the optimal outcome (Wald, 1939). It is one of the core aspects of any financial or investment decision. Traditional finance theory, beginning with MPT, assumes that investors are rational at every point in time (Markowitz, 1959). Further, in this framework, the investor's utility is a function of wealth which is non-decreasing $\left(U^{\prime}(w) \geq 0\right)$ and exhibits diminishing marginal utility $\left(U^{\prime \prime}(w)<0\right)$ - in other words, the investor is risk-averse. However, based on the works of Friedman and Savage (1948), Markowitz (1952a) and Kahneman and Tversky (1979), prospect theory was developed as a contender to the traditional framework. Friedman and Savage (1948) provide a hypothesis which includes the traditional axioms of von Neumann and Morgenstern (1944) as well as a theoretical justification for a section of the utility function to be convex - a section which exhibits increasing marginal utility $\left(U^{\prime \prime}(w)>0\right.$ ). Markowitz (1952a) in an attempt to refine the work of Friedman and Savage (1948) adds that investors possess a utility function which consists of two concave and two 
convex segments. Using this theoretical foundation, Kahneman and Tversky (1979) find experimental evidence that supports the notion of the utility function described previously and formalise the concept of Prospect Theory. The authors conclude that investors maximise the expected value of the function for the convex segment for negative outcomes and the concave segment for positive outcomes. The evolution of utility functions is presented in Figure 1 below. The left-most function corresponds to that used in Markowitz (1959), the middle-left to that used in Friedman and Savage (1948), the middle-right to that used in Markowitz (1952a) and the right-most to that used in Kahneman and Tversky (1979).

Figure 1: Evolution of utility functions (Lopes, 1987)
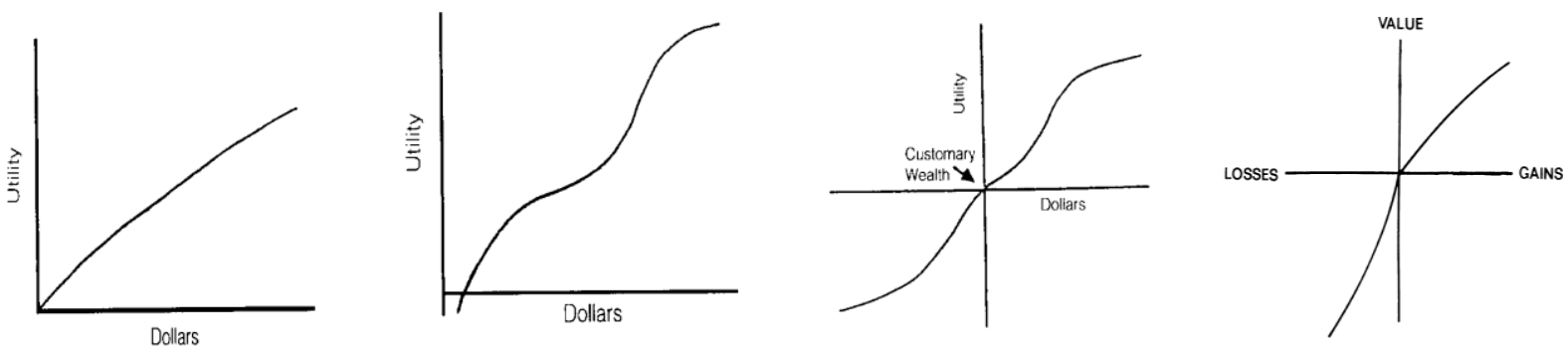

Under the original methodology of Prospect Theory, investors would overweight unlikely events independently of their relative outcomes. This would lead decision makers to choose the worst of two options based on their cumulative probability distributions. Thus the theory as presented in Kahneman and Tversky (1979) gave rise to violations of first order stochastic dominance which presumes an expected utility maximiser possesses an increasing utility function - in other words, the decision maker would instead choose the better of two options based on their cumulative probability distributions. Tversky and Kahneman (1992) thus developed a variant of the original theory, referred to as Cumulative Prospect Theory. Under this variant, cumulative probabilities are transformed to weighted cumulative probabilities, shown in Figure 2 below. This leads extreme events of small probability to be appropriately weighted as opposed to equally weighting all extreme events of small probability (as per the original Prospect Theory). This ensured that first order stochastic dominance was not violated.

\section{Figure 2: A weighting function used in Cumulative Prospect Theory}

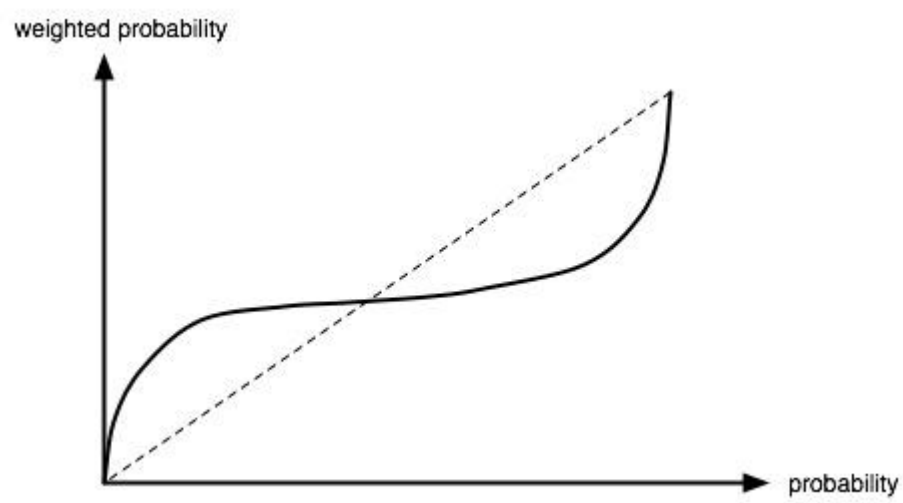

The main development of SD theory was due to Hadar and Russell (1969), Hanoch and Levy (1969), Rothschild and Stiglitz (1970) and Whitmore (1970). Since the development of Prospect Theory, there has been subsequent development into Prospect Stochastic Dominance (Linton, Massoumi \& Whang, 2005). However, application of this technique is beyond the scope of this study. Hadar and Russell (1969) develop theorems for ordering uncertain prospects related to first and second order stochastic dominance. Hanoch and Levy (1969) present a framework for incorporating SD to portfolio selection and optimisation. Rothschild and Stiglitz (1970) view financial returns as a random variable and attempt to model portfolio selection based on this precept and SD rules. Whitmore (1970) develops criterion for third degree dominance and its inclusion in the field of utility theory. Since these developments, application of SD rules to finance, statistics and empirical data have been successful (see Levy, 1992). The primary advantage of SD theory in finance over MPT is that SD theory is based on an axiomatic model of risk-averse preferences. The MPT model of mean-variance optimisation does not model the entire 
spectrum of risk-averse preferences. Moreover, as discussed previously, the use of standard deviation as a measure of risk is not an accurate description of an investor's attitude towards risk. Porter (1974) shows that the use of semi-variance as a measure of risk is consistent with the rules of SD theory. Thus, there exists a relationship between the use of SD rules, semi-variance as a measure of risk and concepts from behavioural finance.

\section{Methodology}

Data: Data was obtained from I-Net Bridge and McGregor BFA. The data consisted of monthly closing prices for three passive indices and select top performing mutual funds in South Africa during the period April 2006 to April 2013. Fund characteristics were obtained from each fund's almanac. We label each fund by a generic alphabetical letter. The sample of funds consists of those that follow popular investment strategies, namely a: value, growth, sector rotation, quantitative, dividend or commodity type strategy.

Statistical methodology: Stochastic Dominance (SD) refers to set of relations which hold between two distributions, characterized by their Cumulative Distribution Functions (CDFs). Consider the CDFs of two functions, $A$ and $B$. If for any argument (or point), $x, \operatorname{CDF}_{\mathrm{A}}(x) \geq \operatorname{CDF}_{\mathrm{B}}(x)$, then $B$ is said to stochastically dominate $A$. At first, the conclusion appears counterintuitive. It is important to note that $\operatorname{CDF}(x)$ represents the proportion of all observations that lie below $x$ - in other words, the area under the curve with a vertical asymptote at $x$. Thus, the CDF which has the greater area under the curve up to and including $x$ has a greater proportion of observations that lie below $x$. This can be further illustrated if $x$ is considered to represent a level of return. If any return below $x$ is considered to be below the minimum acceptable return, then, according to the above relation, $A$ will have a greater proportion of returns that are below the minimum acceptable level. Thus $A$ is dominated by $B$ at first order.

The use of SD in finance circumvents the investigation of distributional properties and yet still presents utility-based interpretations that are economically justifiable. As it is beyond the scope of this study to specify the utility function of the investor who follows the fusion strategy, it is found that ...in the absence of any specification of the utility function, to say that prospect $\mathrm{P}$ is larger than $\mathrm{P}^{\prime}$ in the sense of first order dominance is equivalent to saying that $\mathrm{P}$ is preferred to $\mathrm{P}^{\prime}$ for all monotonic utility functions; and given risk aversion, to say that $\mathrm{P}$ is larger than $\mathrm{P}^{\prime}$ in the sense of second order dominance is equivalent to saying that $\mathrm{P}$ is preferred to $\mathrm{P}^{\prime}$ for all concave utility functions (Hadar \& Russell, 1969, p. 34). Fong, Wong and Lean (2005) are the first to use SD to analyse a momentum strategy. Their primary focus is on the higher orders of SD, due to the compelling utility interpretations these orders contribute. The first three orders of stochastic dominance each have a different interpretation of the utility function of the investor. As such, the concept of proper risk aversion (the precept of first order dominance) and then the higher orders of SD will now be discussed.

Proper risk aversion: "Proper risk aversion is the property that an undesirable lottery can never become desirable by the presence of an independent undesirable lottery" (Pratt and Zeckhauser, 1987). Thus, if an investor is forced to choose between two undesirable outcomes, the outcome not chosen should still remain undesirable, independent of his level of wealth. Utility functions that are monotone obey the above definition. Examples of monotone utility functions include the power and concave exponential utility functions. Consider the following utility function:

$$
U(w)=\int_{0}^{\infty}\left[g(s)-e^{-s w}\right] d F(s)
$$

Where $g$ is an arbitrary function of any non-decreasing value of $s, F$ is non-decreasing and $e^{-s w}$ can be replaced by $w$ when $s=0$. This monotone utility function can be expressed as:

$$
U(w)=U\left(w_{1}\right)+\int_{0}^{\infty}\left[e^{-s w_{1}}-e^{-s w}\right] d F(s)
$$

For any $w_{1}$, where $U\left(w_{1}\right)$ is finite. After differentiating with respect to $w$ and applying Bernstein's Theorem, the following expressions are obtained:

$$
\begin{aligned}
& U^{n}(w)>0 \text { for all } n \geq 1 \text { and } n \text { odd } \\
& U^{n}(w)<0 \text { for all } n \geq 1 \text { and } n \text { even }
\end{aligned}
$$

In other words, the above expressions imply that investors prefer more positively skewed return distributions.

Orders of stochastic dominance: Orders of stochastic dominance can be defined as follows: 


$$
\mathrm{D}^{\mathrm{s}+1}(x)=\int_{0}^{x} \mathrm{D}^{\mathrm{s}}(\mathrm{z}) \mathrm{dz} \text {, for } \mathrm{s}=1,2,3, \ldots
$$

Where $D^{s+1}(x)$ represents a CDF of order $s+1$. If a distribution dominates another at first order, then it is sufficient (yet not necessary) to infer that it will dominate that distribution for any successive order. Definitions are now offered for first, second and third order SD.

Definition 1: First order stochastic dominance (FSD): Let $F$ and $G$ be the cumulative distributions of two risky assets, $x$ be the uncertain ${ }^{1}$ return and $U$ be the utility function. Further, assume that all investors are non-satiated $\left(U^{\prime}(x) \geq 0\right) . F$ is said to dominate $G$ at first order if:

$$
F(x) \leq G(x) \text { for all } x
$$

If the investor picks the asset whose returns are given by $\mathrm{G}$, there is a higher probability he will earn lower returns than if he were to pick the asset whose returns are given by F. In realistic scenarios, FSD is a stringent criterion to rely upon as it does not describe the risk appetite of the investor - only that the investor prefers more wealth to less wealth. In Figure 3 below, option A dominates option B as A has a smaller area under its curve.

Figure 3: First order stochastic dominance

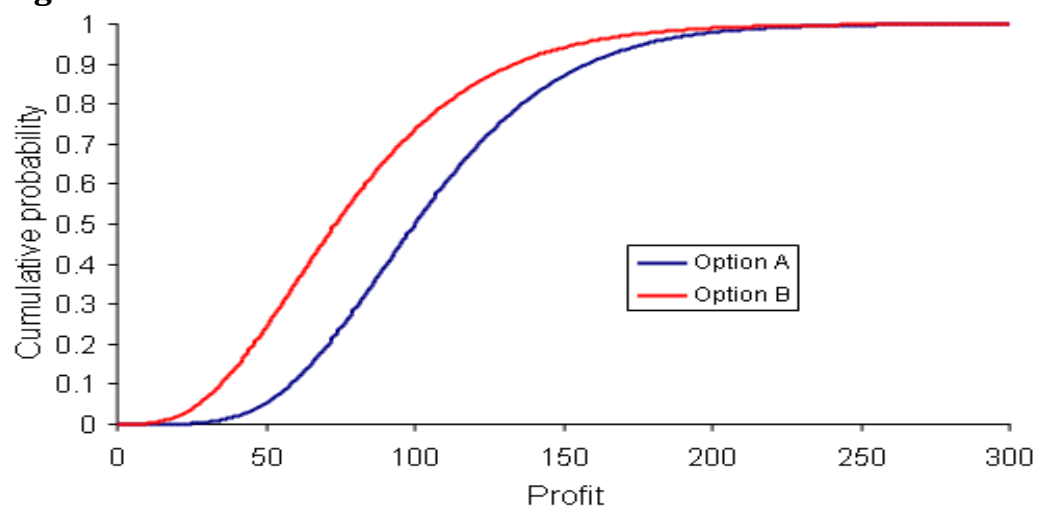

Definition 2: Second order stochastic dominance (SSD): Let $F$ and $G$ be defined as above for Definition 1. Then, $F$ is said to dominate $G$ at second order for all investors with utility functions satisfying $U^{\prime}(x) \geq 0$ and $U^{\prime \prime}(x) \leq 0$ if:

$$
\int_{-\infty}^{x}[G(z)-F(z)] d z \geq 0 \text { for all } x
$$

SSD applies to investors who are non-satiated and risk-averse. In Figure 4 below, option A has second order dominance over option B as function $D(z)$ is positive for all $x$. However, option A does not have first order dominance over option B as A has a larger area under its curve.

Figure 4: Second order stochastic dominance

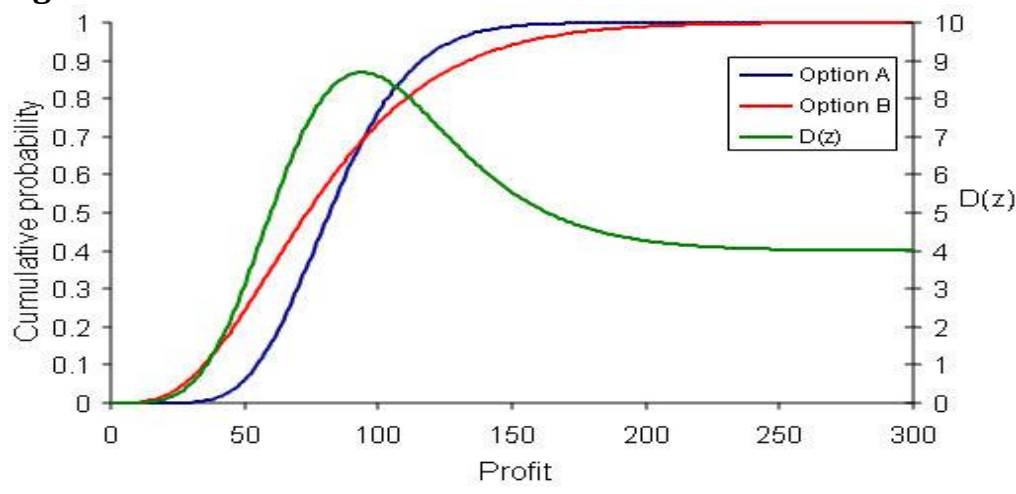

\footnotetext{
${ }^{1}$ Uncertainty refers to both an unknown outcome, $x$, and an unknown distribution of $x$. This is in contrast to risk which refers to an unknown outcome $x$ with a known distribution of $x$ (Knight, 1921). One could argue that financial returns have log-normal distributions but there is no prevailing consensus on this point (see Campbell, Lo and MacKinlay, 1997).
} 
Definition 3: Third order stochastic dominance (TSD): Let $F$ and $G$ be defined as above for Definition 1. Then, $F$ is said to dominate $G$ at third order for all investors with utility functions satisfying $U^{\prime}(x) \geq 0$, $U^{\prime \prime}(x) \leq 0$ and $U^{\prime \prime \prime}(x) \geq 0$ if: $\mathrm{m}_{\mathrm{F}}>\mathrm{m}_{\mathrm{G}}$ and

$$
\int_{-\infty}^{x} \int_{-\infty}^{v}[G(z)-F(z)] d z d v \geq 0 \text { for all } x
$$

Where $m$ denotes expected return. For an investor who is non-satiated, risk-averse and has a decreasing absolute risk aversion, third order SD provides the criterion for ranking returns.

The link to mean-variance analysis: Arguably, the use of standard deviation as measure of risk is tenuous at best. However, in two particular scenarios, the use of standard deviation is acceptable - when returns are normally distributed or when investors have quadratic utility functions. SD analysis relaxes these assumptions and provides a more general framework for analyzing the risk-return framework. Similar to mean-variance analysis, SD analysis provides a means of ranking portfolios but within a less restrictive framework.

\section{Methods of analysing performance}

Stochastic dominance tests: Tests for stochastic dominance are non-parametric (there is no fixed structure of the model used), make no assumptions about the distribution of asset returns and minimal assumptions on investor utility (namely, that the investor prefer more wealth to less). However, it is quite sensitive to outliers in the distribution. The use of stochastic dominance testing allows for variation in the screening protocol. The order of the screens can then be changed to determine if a particular order performs better than another. However, this avenue is left for future research. Linton, Massoumi and Whang (2005) present a generalized procedure for estimating first and second order dominance where observations are allowed to be auto correlated and there is dependence amongst observations. ${ }^{2}$ These relaxations of the independence and identical observations assumptions fit well when returns of different funds are compared in the same market. The authors offer a procedure for estimation that consists of finding at least one observation those results in a strictly positive value. In other words, it searches for that observation which results in the smallest positive area between two graphs. The estimation procedure for first and second order dominance, respectively, is given by:

$$
\begin{gathered}
d^{*}=\min \max [G(z)-F(z)] \\
s^{*}=\min \max \int_{-\infty}^{z}[G(t)-F(t)] d t
\end{gathered}
$$

As such, the hypotheses to be tested are:

- $H_{0}^{d}: \mathrm{d}^{*} \leq 0$ against $H_{1}^{d}: \mathrm{d}^{*}>0$

- $H_{0}^{s}: \mathrm{s}^{*} \leq 0$ against $H_{1}^{s}: \mathrm{s}^{*}>0$

The first hypothesis tests for first order dominance, whilst the second tests for higher order dominance. The critical values for these distributions are obtained via a sub-sampling approach. The results obtained from these smaller samples construct the distribution of possible values for $d^{*}$ and $s^{*}$. Thereafter, using the entire sample, one determines if these values lie at the appropriate significance level in the distribution. ${ }^{3}$ The authors acknowledge that the use of the sub-sampling approach makes their test conservative. These tests are conducted in the statistical software, Model Risk 4.0, by Vose Software (Vose, 2011).

\section{Results}

The tests for stochastic dominance were conducted in Microsoft Excel ${ }^{\circledR}$, using the add-in Model Risk 4.0 by Vose Software (Vose, 2011). This statistical tool is designed to work in a similar manner to other formulae present in Microsoft Excel and provide the user with a final outcome. Using the function, vosedominance, one of three outcomes is possible. The first distribution can show first or second order dominance over the second distribution, or the result could be inconclusive - indicative that perhaps

\footnotetext{
${ }^{2}$ Another common alternative is the test offered by Davidson and Duclous (2000), described in Appendix A.

${ }^{3}$ The interested reader is referred to Linton, Massoumi and Whang (2005) for a detailed discussion.
} 
higher order dominance is possible. These results are also presented in graphical form. It is important to note that whichever strategy is found to be the stochastically dominant one, does not imply that over a longer sample period, the said relationship will hold. Table 1 below shows the results of our stochastic dominance tests. It reports the order at which the mutual fund dominates the corresponding passive index. For example, Fund $\mathrm{C}$ dominates the Small Cap Index at $2^{\text {nd }}$ order, implying that a risk adverse investor would prefer Fund C over the Small Cap Index. An inconclusive result shows that there is neither $1^{\text {st }}, 2^{\text {nd }}$ nor $3^{\text {rd }}$ order dominance, implying that dominance at higher orders is possible. However, higher order dominance is often difficult to interpret and provides little insight to the risk appetite of the investor.

Table 1: Stochastic Dominance Results

\begin{tabular}{llll}
\hline & Small Cap Index & ALSI & Top 40 Index \\
\hline Fund A & 3 & 3 & 2 \\
Fund B & 2 & 2 & 3 \\
Fund C & 2 & 1 & 2 \\
Fund D & 1 & Inconclusive & 2 \\
Fund E & 1 & Inconclusive & 3 \\
Fund F & 2 & Inconclusive & 2 \\
Fund G & 2 & 1 & 3 \\
Fund H & 2 & 2 & 2 \\
Fund I & 3 & 3 & 2 \\
Fund J & 2 & 2 & 2 \\
Fund K & 2 & 2 & 2 \\
\hline
\end{tabular}

Without any further information, it can be seen that the investor who simply prefers more wealth to less will choose Fund C or Fund G over the ALSI (the market index). The results thus broadly indicate that over our sample period of 2006 to 2013, mutual funds have been preferred over lower risk passive market indices by investors who are generally risk averse. While this may appear counter intuitive, recall that first order stochastic dominance relies on an investor preferring more wealth to less, and says little about the investor's risk aversion. Second order however refers to an investor who is risk averse. We now turn our attention to the characteristics of each mutual fund and evaluate whether the investor's choice given by stochastic dominance corresponds to the risk profile set out by the fund. This comparison is shown in Table 2 below. The risk profile of each mutual fund was obtained by its respective prospectus. The typical investor would investigate this fund sheet to determine if the risk and return profile of the fund is in line with his expectations. We compare the risk profile from the fund sheet to three passive market indices (the Small Cap Index, Top 40 Index and All Share Index) via stochastic dominance. It is a priori expected that the implications of the stochastic dominance results would be in line with the risk profile from the fund sheet - in other words, if the fund is advertised as high risk, then a $3^{\text {rd }}$ order dominance result (or higher) is expected.

Table 2 below provides a summary of these results. The "success rate" is simply a count of where the risk profile advertised is in line with the results from stochastic dominance. For example, Fund B is advertised as medium risk, thus we would expect that $2^{\text {nd }}$ order dominant investors (those that are risk averse), at the very least, to invest in the fund. It is acknowledged that a risk averse investor may not wish to invest in any fund; however, we reserve this investor profile to those that are first order dominant - those that simply prefer more wealth to less. It is found that of the 11 mutual funds examined; only 4 attract the correct type of investor, 3 attract a mixture of investors and 4 attract (arguably) the wrong type of investor. 
Table 2: Comparison of risk profiles to results from stochastic dominance tests

\begin{tabular}{|c|c|c|c|c|c|c|}
\hline \multirow[t]{2}{*}{ Fund } & \multirow{2}{*}{\multicolumn{2}{|c|}{$\begin{array}{l}\text { Risk Profile } \\
\text { as } \\
\text { advertised }\end{array}$}} & \multirow{2}{*}{$\begin{array}{l}\text { SD Result relative } \\
\text { to Small Cap } \\
\text { Index }\end{array}$} & \multirow{2}{*}{$\begin{array}{l}\text { SD Result } \\
\text { relative to ALSI }\end{array}$} & \multirow{2}{*}{$\begin{array}{l}\text { SD } \\
\text { relative to Tosult } \\
40 \text { Index }\end{array}$} & \multirow[t]{2}{*}{ Success rate } \\
\hline & & & & & & \\
\hline A & High & & DRA & DRA & RA & $66 \%$ \\
\hline B & Medium & & RA & RA & DRA & $66 \%$ \\
\hline $\mathrm{C}$ & Low & & RA & More wealth & RA & $33 \%$ \\
\hline D & $\begin{array}{l}\text { Medium } \\
\text { High }\end{array}$ & to & More wealth & Inconclusive & RA & $0 \%$ \\
\hline $\mathrm{E}$ & $\begin{array}{l}\text { Low } \\
\text { Medium }\end{array}$ & to & More wealth & Inconclusive & DRA & $33 \%$ \\
\hline $\mathrm{F}$ & $\begin{array}{l}\text { Low } \\
\text { Medium }\end{array}$ & to & RA & Inconclusive & DRA & $33 \%$ \\
\hline G & $\begin{array}{l}\text { Low } \\
\text { Medium }\end{array}$ & to & RA & More wealth & DRA & $66 \%$ \\
\hline $\mathrm{H}$ & $\begin{array}{l}\text { Medium } \\
\text { High }\end{array}$ & to & RA & RA & RA & $100 \%$ \\
\hline I & $\begin{array}{l}\text { Medium } \\
\text { High }\end{array}$ & to & DRA & DRA & RA & $100 \%$ \\
\hline $\mathrm{J}$ & $\begin{array}{l}\text { L:ow } \\
\text { Medium }\end{array}$ & to & RA & RA & RA & $100 \%$ \\
\hline K & $\begin{array}{l}\text { Medium } \\
\text { High }\end{array}$ & to & RA & RA & RA & $100 \%$ \\
\hline
\end{tabular}

*Note: "More Wealth" refers to $1^{\text {st }}$ order dominance; RA refers to Risk Aversion (2nd order dominance) and DRA refers to Decreasing Risk Aversion ( $3^{\text {rd }}$ order dominance).

\section{Conclusion}

Our study investigated the choice of stochastic dominance in determining the risk profiles of investors who invest in mutual funds over a passive market index. While our results are somewhat mixed, it provides insight into investor behaviour over our sample period of April 2006 to April 2013. Over this time frame, one would expect an investor's risk appetite to have decreased as a result of the financial crisis of 2008 to 2009. Indeed, the results presented above can be extended to cover a complete market cycle and further segmented into different phases of the market cycle. The results of this study can be extended into industry practice where the advertised profiles of mutual funds can be possibly reexamined based on the type of investor currently attracted. Further, it is worth examining the reason as to why the investor with a particular risk profile would choose to invest in a mutual fund outside of his preferences. Concepts from the field of behavioural finance could assist in this regard.

\section{References}

Davidson, R. \& Duclous, J. Y. (2000). Statistical Inference for Stochastic Dominance and for the Measurement of Poverty and Inequality. Econometrica, 68, 1435-1464.

Fama, E. (1965). Random Walks in Stock Market Prices. Financial Analysts Journal , 21(5), 55-59.

Fong, W., Wong, W. \& Lean, H. (2005). International momentum strategies: a stochastic dominance approach. Journal of Financial Markets, 8(1), 89-109.

Friedman, M. \& Savage, L. (1948). The Utility of Choices Involving Risk. Journal of Political Economy, 56 (4), 279-304.

Hadar, J. \& Russell, W. (1969). Rules for Ordering Uncertain Prospects. American Economic Review, 59, $25-$ 34.

Hanoch, G. \& Levy, H. (1969). The Efficiency Analysis of Choices Involving Risk. Review of Economic Studies , 36, 335-346.

Kahneman, D. \& Tversky, A. (1979). Prospect Theory: An Analysis of Decision Under Risk. Econometrica , 47(2), 263-291.

Kendall, M. (1953). The Analysis of Time Series Economics Part 1: Prices. Journal of the Royal Statistical Society, 116(1), 11-34.

Levy, H. (1992). Stochastic Dominance and Expected Utility: Survey and Analysis. Journal of Management Science, 38(4), 555-593. 
Linton, O., Massoumi, E. \& Whang, Y. (2005). Consistent Testing for Stochastic Dominance: A Subsampling Approach. Review of Economic Studies, 72(3), 735-765.

Lo, A. (2004). Reconciling Efficient Markets with Behavioral Finance: The Adaptive Markets Hypothesis. Journal of Investment Consulting, 7(1), 21-44.

Lo, A. (2005). The Adaptive Markets Hypothesis: Market Efficiency from an Evolutionary Perspective. Journal of Portfolio Management, 30(1), 15-29.

Lopes, L. (1987). Between Hope and Fear: The Psychology of Risk. Advances in Experimental Social Psychology, 20, 255-295.

Malkiel, B. (2005). Reflections on the Efficient Markets Hypothesis: 30 Years Later. Financial Review , 40, $1-9$.

Markowitz, H. (1952a). The Utility of Wealth. Journal of Political Economy , 60, 151-156.

Markowitz, H. (1959). Portfolio Selection: Efficient Diversification of Investments. John Wiley and Sons.

Porter, R. (1974). Semi Variance and Stochastic Dominance: A Comparison. American Economic Review, 64, 200-204.

Pratt, J. \& Zeckhauser, R. (1987). Proper Risk Aversion. Econometrica, 55, 143-154.

Roberts, H. (1959). Stock Market "Patterns" and Financial Analysis: Methodological Suggestions. Journal of Finance, 14(1), 1-10.

Rothschild, M. \& Stiglitz, J. (1970). Increasing Risk: 1. A Definition. Journal of Theory , 2, 225-243.

Seetharam, Y. \& Auret, C. (2013). Fusion Investing: An esoteric approach to portfolio formation. Unpublished.

Sharpe, W. (1964). Capital asset prices: A theory of market equilibrium under conditions of risk. Journal of Finance, 19(3), 425-442.

Todea, A., Ulici, M. \& Silaghi, S. (2009). The Adaptive Markets Hypothesis: Evidence from Asia-Pacfic Financial Markets, Review of Finance and Banking , 2, 7-13

Tversky, A. \& Kahneman, D. (1992). Advances in Prospect Theory: Cumulative Representations of Uncertainty. Journal of Risk and Uncertainty , 5, 297-323.

von Neumann, J. \& Morgenstern, O. (1944). Theory of Games and Economic Behaviour. Princeton University Press.

Vose. (2011). Model Risk 4.0. Belgium.

Wald, A. (1939). Contributions to the Theory of Statistical Estimation and Testing Hypotheses. Annals of Mathematical Statistics , 10(4), 299-326.

Whitmore, G. (1970). Third Degree Stochastic Dominance. American Economic Review , 60, 457-459.

\section{Appendix A}

This Appendix describes an alternative test for stochastic dominance developed by Davidson and Duclous (2000). It is important to note that as both the Linton et al. (2005) method as well as the test described here are valid, the choice is determined by the data to be analysed. The Davidson and Duclous (DD) (2000) test for stochastic dominance that is applicable to both independent and dependent samples from a joint distribution. The test compares the cumulative distribution functions over a grid of points. A potential caveat of the test is that the number of grid points is chosen arbitrarily and the consistency of the test statistic is affected by the use of a finite grid. The test is outlined in detail below.

The following hypotheses are tested:

1. $\mathrm{H}_{0}: \mathrm{D}_{\mathrm{W}}^{\mathrm{s}}\left(\mathrm{x}_{\mathrm{k}}\right)=\mathrm{D}_{\mathrm{L}}^{\mathrm{s}}\left(\mathrm{x}_{\mathrm{k}}\right) \forall \mathrm{x}_{\mathrm{k}}, \mathrm{k}=1 \ldots \mathrm{K}$

2. $\mathrm{H}_{\mathrm{A}}: \mathrm{D}_{\mathrm{W}}^{\mathrm{S}}\left(\mathrm{x}_{\mathrm{k}}\right) \neq \mathrm{D}_{\mathrm{L}}^{\mathrm{s}}\left(\mathrm{x}_{\mathrm{k}}\right)$ for some $\mathrm{x}_{\mathrm{k}}$

3. $\mathrm{H}_{\mathrm{A} 1}: \mathrm{D}_{\mathrm{W}}^{\mathrm{s}}\left(\mathrm{x}_{\mathrm{k}}\right)>\mathrm{D}_{\mathrm{L}}^{\mathrm{s}}\left(\mathrm{x}_{\mathrm{k}}\right)$ for some $\mathrm{x}_{\mathrm{k}}$

$4 . \mathrm{H}_{\mathrm{A} 2}: \mathrm{D}_{\mathrm{W}}^{\mathrm{s}}\left(\mathrm{x}_{\mathrm{k}}\right)<\mathrm{D}_{\mathrm{L}}^{\mathrm{s}}\left(\mathrm{x}_{\mathrm{k}}\right)$ for some $\mathrm{x}_{\mathrm{k}}$

Davidson and Duclous (2000) construct the following sample statistics, where to avoid notation clutter; the grid index $k$ is suppressed for each statistic:

$$
\begin{aligned}
\widehat{D}_{W}^{S}(x) & =\frac{1}{N(s-1) !} \sum_{i=1}^{N}\left(x_{k}-W_{i}\right)^{s-1}+ \\
\widehat{D}_{L}^{S}(x) & =\frac{1}{N(s-1) !} \sum_{i=1}^{N}\left(x_{k}-L_{i}\right)^{s-1}+
\end{aligned}
$$




$$
\begin{gathered}
\hat{V}_{W}^{S}=\frac{1}{N}\left[\frac{1}{N((s-1) !)^{2}} \sum_{i=1}^{N}\left(x_{k}-W_{i}\right)_{+}^{2(s-1)}-\widehat{D}_{W}^{S}(x)^{2}\right] \\
\hat{V}_{L}^{S}=\frac{1}{N}\left[\frac{1}{N((s-1) !)^{2}} \sum_{i=1}^{N}\left(x_{k}-L_{i}\right)_{+}^{2(s-1)}-\widehat{D}_{L}^{S}(x)^{2}\right] \\
\widehat{V}_{W, L}^{S}=\frac{1}{N}\left[\frac{1}{N((s-1) !)^{2}} \sum_{i=1}^{N}\left(x_{k}-W_{i}\right)_{+}^{S-1}\left(x_{k}-L_{i}\right)_{+}^{S-1}-\widehat{D}_{W}^{S}(x) \widehat{D}_{L}^{S}(x)\right] \\
\hat{V}^{S}(x)=\hat{V}_{W}^{S}(x)+\widehat{V}_{L}^{S}(x)-2 \widehat{V}_{W, L}^{S}(x)
\end{gathered}
$$

Consider the t-statistic:

$$
T^{S}(x)=\frac{\widehat{D}_{W}^{S}(x)-\widehat{D}_{L}^{S}(x)}{\sqrt{\widehat{V}^{S}(x)}}
$$

Under the null hypothesis, $T^{S}(x)$ is asymptotically distributed as a standard normal variate. To implement the DD test, we can compute a t-statistic at each grid point and reject the null hypothesis if the largest $\mathrm{t}$ statistic is significant. As suggested by Davidson and Duclous (2000), the joint test size can be controlled by using critical values of the studentised maximum modulus (SMM) distribution in place of the normal distribution. Let $M_{\infty}^{k}(x)$ denote the (1- $a$ ) percentile of the SMM statistic with $k$ and infinite degrees of freedom. Then, the following decision rules can be used:

1. If $\left|T^{s}\left(x_{k}\right)\right|<M_{\infty, a}^{k}$ for $k=1, \ldots, K$ then accept $H_{0}$

2.If $T^{s}\left(x_{k}\right)<M_{\infty, a}^{k}$ for all $k$ and $-T^{s}\left(x_{k}\right)>M_{\infty, a}^{k}$ for some $k$ then accept $H_{A 1}$

3. If $-T^{s}\left(x_{k}\right)<M_{\infty, a}^{k}$ for all $k$ and $T^{s}\left(x_{k}\right)>M_{\infty, a}^{k}$ for some $k$ then accept $H_{A 2}$

4. If $T^{s}\left(x_{k}\right)>M_{\infty, a}^{k}$ for some $k$ and $-T^{s}\left(x_{k}\right)>M_{\infty, a}^{k}$ for some $k$ then accept $H_{A}$

In empirical studies, the number of grid points is usually chosen based on rules of thumb. Barrett and Donald (2003) show that for reasonably large samples (greater than 500 observations), the DD test works well for $K=10$. Actual applications may require a finer grid because as Barrett and Donald (2003, p. 91) point out, a coarse grid may miss out important differences in the distributions. The $5 \%$ asymptotic critical value is 3.254 from Stoline and Ury (1979). 